\title{
An Integrated Dynamic Market Mechanism for Real-time Markets and Frequency Regulation
}

\author{
Dylan Shiltz, Student, IEEE, Miloš Cvetković, Member, IEEE, and Anuradha Annaswamy, Fellow, IEEE
}

\begin{abstract}
The intermittent and uncertain nature of renewables represents a major challenge for large scale adoption of sustainable energy resources. Of particular concern is the need to maintain both quality of grid frequency and low costs of regulation reserves in the face of increasing fluctuations in renewables. To this end, we propose an integrated dynamic market mechanism (DMM) which combines real-time market and frequency regulation allowing market players, including renewable generators and flexible consumers, to iteratively negotiate electricity prices at the wholesale level while using the most recent information on the available wind power and the quality of grid frequency. Main features of the integrated DMM are (i) a Newton-Raphson-based method which leads to fast convergence to the optimal dispatch, (ii) use of an aggregated frequency error as a feedback signal for the negotiation process which leads to reduced regulation capacity requirements. The benefits of this DMM are illustrated via simulations on the IEEE 118 bus system.
\end{abstract}

Index Terms-Dynamic market mechanism, real-time market, regulation requirements, automatic generation control, area control error

\section{NOMENCLATURE}

$D_{r} \quad$ Set of flexible consumers

$G_{c} \quad$ Set of conventional generators

$G_{r} \quad$ Set of renewable generators

$\phi_{n} \quad$ Set of consumer indices at node $n$

$\theta_{n} \quad$ Set of conventional generator indices at node $n$

$\vartheta_{n} \quad$ Set of renewable generator indices at node $n$

$\Omega_{n} \quad$ Set of nodes adjacent to node $n$

$B_{n m} \quad$ Susceptance of the line $n$ to $m$

$\bar{P}_{n m} \quad$ Maximum capacity of the line $n$ to $m$

$V \quad$ Set of all network nodes

$B_{\text {line }}$ Diagonal line susceptance matrix

$A \quad$ Node incidence matrix

$A_{r} \quad$ Reduced order node incidence matrix

$A_{D r} \quad$ Demand response incidence matrix

$A_{G c} \quad$ Conventional generator incidence matrix

$A_{G r} \quad$ Renewable generator incidence matrix

$J_{e q} \quad$ Equivalent inertia of a balancing area

\section{INTRODUCTION}

Increasing penetration of renewable energy resources (RER), motivated by sustainability concerns, introduces challenges across all aspects of power system planning and operations. The primary challenge presented by significant

All authors are with the Department of Mechanical Engineering, Massachusetts Institute of Technology, Cambridge, MA, 02139 USA e-mail: \{djshiltz,mcvetkov,aanna\}@mit.edu

This work was supported by the NSF initiative, Award no. EFRI-1441301. renewable penetration is their intermittency. As there can be a substantial discrepancy between the predicted and actual renewable generation in the system [1], the grid requires a large number of fast-ramping reserves, usually provided by natural gas-fired power plants [2]. However, as the penetration of renewables continues to rise, these reserve requirements may become prohibitively expensive [3]. Direct energy storage based solutions [4]-[6] are still economically unjustifiable, and thus, currently absent at the grid-scale level.

The use of demand response (DR) is a popular approach suggested of late to address intermittency and uncertainty in renewable generation. Real-time pricing has been widely viewed as the key to large scale implementation of demand response (see [7] for an overview). This usually involves scheduling consumption during periods of low prices to minimize costs to consumers or to optimize grid level operations. As there may be an inherent uncertainty in allowing consumers to react to price, stability of the grid may be compromised and can lead to a volatile market [8], [3]. In these papers and others that have dealt with DR, the framework is often one where any DR-compatible consumers act as price takers; that is, the consumers react to prices after they are settled instead of actively participating in the wholesale market.

In addition to market level solutions, enhancing current frequency regulation practice is crucial for accommodating high penetration of renewable power. Frequency regulation is necessitated by imbalances in power that occur between any two market clearing instances caused due to unpredictable inflexible demand and/or intermittent RERs. This correction is typically implemented through the use of automatic generation control (AGC) in response to the error in grid frequency [9]. As renewable penetration increases, new integrated solutions that address both the market-level needs and the AGC needs are warranted and are being proposed [10]-[12]. Reference [10] proposes a hierarchical approach for combining economic dispatch and regulation based on the disturbance segmentation. Reference [11] proposes frequency aware economic dispatch for handling significant grid frequency errors. More recently, reference [12] proposes modifications to the AGC which allow a better integration with the OPF problem.

This paper proposes an alternative market structure that has the ability to efficiently embrace DR and AGC, and accommodates intermittency and uncertainty that are concomitant in renewable generation, leading to efficient integration of renewables at the market level, and reduced regulation requirements at the AGC level. This market structure consists of an integrated dynamic market mechanism (DMM) and builds on the DMM framework proposed in [13]-[16]. This DMM 
proposes that DR-compatible consumers act as price setters, by playing an active role in the electricity market leading towards financially binding agreement involving quantity and price. It consists of a distributed decision making process in which market players and the ISO iteratively exchange information until an optimal generation/consumption dispatch is reached. The iterative approach in DMM allows the incorporation of any real-time information that becomes available about the intermittent RER, thereby having the potential to generate higher social welfare when compared to OPF [16]. In [14], integration with the AGC timescales is explored and conditions under which the resulting hierarchical transactive structure can achieve an improved performance is explored.

The DMM proposed in [13]-[16] however suffers from two major deficiencies: (1) the speed of convergence of the underlying gradient method imposes significant limitations on the rate of convergence and therefore the requisite market clearing period; (2) the impact of the DMM on faster timescales for frequency regulation and AGC is not addressed. In this paper, we address both of these deficiencies.

We propose an integrated DMM in this paper that combines market clearing procedure with automatic generation control and allows for highly efficient and timely operation of power systems with large penetration of intermittent RER power. The integrated DMM is derived in two steps. In the first step, we propose the use of a modified Newton-Raphson method which ensures significantly faster convergence and therefore a shorter market clearing period compared to that of the OPF. Additionally, the proposed method utilizes a minimum information exchange while sensitive information, such as generation/consumption bounds and cost/utility curves, remain private to producers/consumers. In the second step, we propose the use of an aggregated grid frequency error as a feedback signal from the frequency regulation layer to the market layer for improving optimal dispatch. The resulting integrated DMM is shown to lead to better utilization of renewables at the market-level and lower regulation capacity and energy requirements at the AGC level.

In Section II, we outline the DMM algorithm, give conditions for provable convergence and discuss the real-time implementation. In Section III, we describe the integrated DMM which combines real-time market and frequency regulation in order to correct market dispatches. In Section IV simulation results are presented. Section V provides concluding remarks.

\section{Proposed Electricity Market Model}

In this section, we present the first step in our integrated market design, which corresponds to DMM. Following preliminaries that present the overall time scales, the framework for market clearing is presented in Section II-B. This is followed by the actual DMM in II-C, its convergence properties in II-D, wind model in II-E, and implementation in II-F.

\section{A. Preliminaries}

We denote $x^{i}$ as the value of quantity $x$ at time $t_{i}$ (see Fig. 1). We then denote four different time-scales, $t_{k}, t_{K}, t_{m}$, and $t_{M}$, with corresponding periods $T_{k}, T_{K}, T_{m}, T_{M}$, where

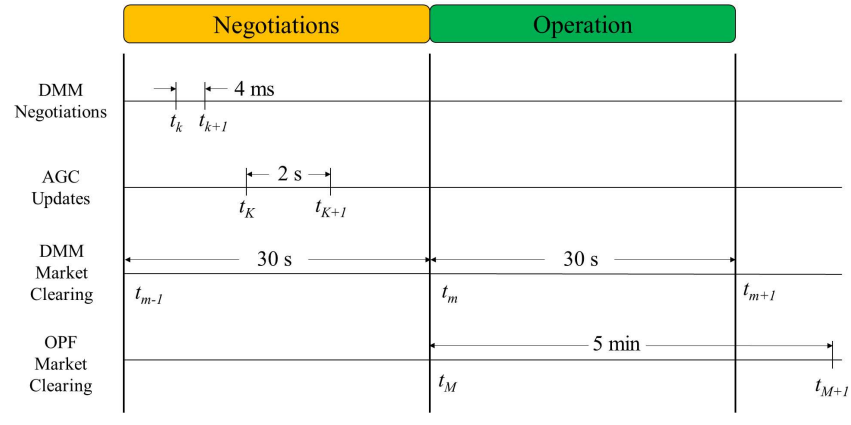

Fig. 1. Underlying time-scales in the proposed DMM.

$T_{\ell}=t_{\ell+1}-t_{\ell}$ for time-scale $\ell$. The periods $T_{K}$ and $T_{M}$ correspond to the existing time-scales associated with AGC dispatch and real-time market clearing time (and are typically $2 \mathrm{sec}$ and $5 \mathrm{~min}$, respectively). The periods $T_{k}$ and $T_{m}$ are introduced to define our integrated DMM, where $T_{k} \ll T_{K}$ and $T_{K} \ll T_{m} \ll T_{M}$, and will be defined more precisely later. Finally, we define index sets $\Phi_{m}, \Phi_{M}, \Psi_{m}$, and $\Psi_{M}$, where $\Phi_{\ell}=\left\{K \mid t_{K}<t_{\ell}\right\}$ and $\Psi_{\ell}=\left\{K \mid t_{\ell} \leq t_{K}<t_{\ell+1}\right\}$.

\section{B. Economic Dispatch Framework with Barrier Functions}

In order to describe the DMM, we start with market clearing, the procedure by which economic dispatch is carried out [16]. Participants in this market are generation companies (GenCos) that seek to recover their fuel costs, consumer companies (ConCos) who seek to procure electric energy for their needs, and ISO who acts on behalf of ConCos and GenCos, maximizing the utility of ConCos while minimizing the cost of GenCos. The objective function that ISO uses to solve this optimization problem is commonly termed Social Welfare, denoted here by $S_{W}$ and defined as

$S_{W}=\sum_{i \in D r} U_{D r_{i}}\left(P_{D r_{i}}\right)-\sum_{i \in G c} C_{G c_{i}}\left(P_{G c_{i}}\right)-\sum_{i \in G r} C_{G r_{i}}\left(P_{G r_{i}}\right)$

where quadratic utility curves of flexible consumers and quadratic cost curves of conventional and renewable generators are given in (1), (2) and (3), respectively.

$$
\begin{aligned}
& U_{D r_{i}}\left(P_{D r_{i}}\right)=b_{D r_{i}} P_{D r_{i}}+\frac{c_{D r_{i}}}{2} P_{D r_{i}}^{2} \\
& C_{G c_{i}}\left(P_{G c_{i}}\right)=b_{G c_{i}} P_{G c_{i}}+\frac{c_{G c_{i}}}{2} P_{G c_{i}}^{2} \\
& C_{G r_{i}}\left(P_{G r_{i}}\right)=b_{G r_{i}} P_{G r_{i}}+\frac{c_{G r_{i}}}{2} P_{G r_{i}}^{2}
\end{aligned}
$$

Parameters $b_{\ell}$ and $c_{\ell}$ in the above equations reflect base and incremental cost-utility parameters, respectively, while $P_{D r_{i}}$ is the power demand of flexible consumers and $P_{G c_{i}}$ and $P_{G r_{i}}$ is the power supplied by conventional and renewable generators. The overall market clearing can then be written as the following optimization problem.

$$
\min -S_{W}
$$

subject to

$$
\begin{array}{r}
P_{D c_{n}}+\sum_{i \in \phi_{n}} P_{D r_{i}}-\sum_{i \in \theta_{n}} P_{G c_{i}}-\sum_{i \in \vartheta_{n}} P_{G r_{i}} \\
+\sum_{m \in \Omega_{n}} B_{n m}\left(\delta_{n}-\delta_{m}\right)=0 \quad \forall n \in V
\end{array}
$$




$$
\begin{array}{rlrl}
\underline{P}_{D r_{i}} \leq P_{D r_{i}} \leq \bar{P}_{D r_{i}} & \forall i \in D_{r} & \\
\underline{P}_{G c_{i}} \leq P_{G c_{i}} \leq \bar{P}_{G c_{i}} & \forall i \in G_{c} & \\
\underline{P}_{G r_{i}} \leq P_{G r_{i}} \leq \hat{\bar{P}}_{G r_{i}} & \forall i \in G_{r} & \\
B_{n m}\left(\delta_{n}-\delta_{m}\right) \leq \bar{P}_{n m} & \forall n \in V, \quad \forall m \in \Omega_{n}
\end{array}
$$

In this problem, prediction of inflexible power consumption at node $n$ is denoted by $P_{D c_{n}}$ and $\delta_{n}$ denotes the voltage angle at node $n$. One of the nodes must be designated as the reference node, at which the voltage angle is defined to be zero. This is a typical assumption for finding a unique solution for power flow problems, also known as the slack bus. Since the true wind generation is not known beforehand, based on wind predictions, an estimate $\hat{\bar{P}}_{G r_{i}}$ is used in (8).

The optimization problem is then solved to compute solution vector $x=\left[\begin{array}{llll}\delta^{T} & P_{D r}^{T} & P_{G c}^{T} & P_{G r}^{T}\end{array}\right]^{T}$, where all of the variables comprising vector $x$ are themselves vectors, e.g. $\delta=\left[\delta_{1}, \ldots, \delta_{n}\right]^{T}$. Throughout this paper we assume vector notation by omitting the corresponding index.

One can accommodate the inequality constraints through the use of barrier functions [17]. For this purpose, we express the constraints in (6)-(8) as $g_{1 i}(x) \geq 0, i \in D_{r}, G_{c}, G_{r}$, and note that these constraints stem from GenCos and ConCos. We express the constraints in (9) as $g_{2 j}(x) \geq 0, j \in V$, which stem from the transmission system limitations. We now define barrier functions $\beta_{1}(x)$ and $\beta_{2}(x)$ as

$$
\beta_{1}(x)=\sum_{i \in D_{r}, G_{c}, G_{r}} \frac{\nu}{g_{1 i}(x)}, \quad \beta_{2}(x)=\sum_{j \in V} \frac{\nu}{g_{2 j}(x)}
$$

where $\nu$ is a small positive constant used to adjust the slope of the barrier. Using these barrier functions, which penalize the solution as it approaches the boundaries of the constraints in (6)-(9), we construct a modified cost function of the form

$$
f(x)=-S_{W}(x)+\beta_{1}(x)+\beta_{2}(x) .
$$

Using the modified cost function (11), and denoting (5) as

$$
h(x)=P_{D c}+\left[A^{T} B_{\text {line }} A_{r} A_{D r}-A_{G c}-A_{G r}\right] x=0
$$

the underlying optimization problem becomes

$$
\begin{aligned}
& \min f(x) \\
& \text { st }: h(x)=0 .
\end{aligned}
$$

Transmission system parameter matrices $A, A_{r}$ and $B_{\text {line }}$ in (12), defined in nomenclature, are a part of a standard DC power flow representation in matrix form [18]. Parameter matrices $A_{D r}, A_{G c}$ and $A_{G r}$ are incidence matrices used to map generation and consumption to their respective nodes.

The Lagrangian of the optimization problem can be expressed as

$$
\mathcal{L}(x, \lambda)=f(x)+\sum_{n \in V} \lambda_{n} h_{n}(x)
$$

The typical procedure in the OPF method is to find the optimal solution $x$ and the corresponding Lagrangian multipliers $\lambda_{n}$ to determine the optimal dispatch and location marginal price.

In today's industry, the market clearing procedure is carried out periodically with period $T_{M}$ (see Fig. 1). In the following subsection, we propose a dynamic market mechanism which clears the market with a shorter period $T_{m}$.

\section{Derivation of DMM}

The optimization problem in (4)-(9) is addressed in the DMM through continued negotiations between generators, consumers, and ISO, with period $T_{k}$ and is described below. For this purpose, Lagrangian (14) of problem (13) could be solved using a first order gradient interior point method as in [16]. Due to slow convergence of such methods, we solve the same optimization problem using a modified version of the Newton-Raphson method. Second order methods, such as this one, require fewer iterations to converge, and thus, are more suitable for time-critical operations.

To apply Newton-Raphson method, we compute the Hessian and gradient of the Lagrangian as

$$
\begin{aligned}
\nabla^{2} \mathcal{L}\left(x^{k}, \lambda^{k}\right) & =\left[\begin{array}{cc}
H^{k} & N^{k} \\
N^{k^{T}} & 0
\end{array}\right] \\
\nabla \mathcal{L}\left(x^{k}, \lambda^{k}\right) & =\left[\begin{array}{c}
\nabla_{x} \mathcal{L}\left(x^{k}, \lambda^{k}\right) \\
h\left(x^{k}\right)
\end{array}\right]
\end{aligned}
$$

where $H^{k}=\nabla_{x x}^{2} \mathcal{L}\left(x^{k}, \lambda^{k}\right)$ and $N^{k}=\nabla h\left(x^{k}\right)$. It follows by inspection that $N^{k}=N$, a constant, for all $k$. To ensure that the Hessian remains non-singular, the Hessian $H^{k}$ is modified to $\bar{H}^{k}=H^{k}+c N N^{T}$ where $c$ is a positive scalar chosen so that $\bar{H}^{k}$ is positive definite [17]. This results in a DMM given by

$$
\begin{aligned}
& x^{k+1}=x^{k}-\alpha \cdot \bar{H}^{k^{-1}} \nabla_{x} \mathcal{L}\left(x^{k}, \hat{\lambda}^{k+1}\right) \\
& \lambda^{k+1}=\hat{\lambda}^{k+1}-\alpha \cdot c \cdot h\left(x^{k}\right)
\end{aligned}
$$

where

$$
\hat{\lambda}^{k+1}=\left(N^{T} \bar{H}^{k^{-1}} N\right)^{-1}\left(h\left(x^{k}\right)-N^{T} \bar{H}^{k^{-1}} \nabla f\left(x^{k}\right)\right)
$$

and $\alpha$ is a suitably chosen step size.

\section{Convergence of DMM}

In this section we will show that iterates (17)-(19) converge to the unique optimum of problem (13) for a sufficiently small step size $\alpha$.

Theorem 1: If $\bar{H}$ is positive definite and the initial state $x_{0}$ is strictly feasible (i.e. $g_{1 i}(x)>0, i \in D_{r}, G_{c}, G_{r}$ and $\left.g_{2 j}(x)>0, j \in V\right)$, there exists a step size $\alpha$ such that the DMM iterates (17)-(19) converge to the unique global optimum of problem (13).

Proof: We prove Theorem 1 using the following two steps:

Step 1: Problem (13) is strictly convex. Step 1 follows because the equality constraints are affine and the objective function is strictly convex over the domain of interest, since the cost curves of the GenCo's are strictly convex, the utility curves of ConCo's are strictly concave, and the barrier functions are strictly convex for $g(x)>0$. This also implies that KKT conditions [17] are sufficient for global optimality.

Step 2: There exists a step size $\alpha$ such that the sequence $x^{k}$ converges to a stationary point of $\mathcal{L}(x, \lambda)$. Step 2 can be proved as follows. We rewrite the iterates from (17) as

$$
x^{k+1}=x^{k}-\alpha \cdot \bar{H}^{k^{-1}} \nabla_{x} \mathcal{L}_{c}\left(x^{k}, \lambda^{k+1}\right)
$$

where $\mathcal{L}_{c}$ is the Lagrangian of the problem of minimizing $f(x)+\frac{c}{2}\|h(x)\|^{2}$ subject to $h(x)=0$. For all feasible points 
(i.e. for all $x$ such that $h(x)=0$ ), these are identical problems, and so their minima coincide. Next we require that $\nabla \mathcal{L}_{c}$ is Lipschitz continuous, or that

$$
\left\|\nabla \mathcal{L}_{c}(u)-\nabla \mathcal{L}_{c}(v)\right\| \leq L\|u-v\|, \quad \forall u, v \in \mathcal{X}
$$

holds for some $L>0$, where $\mathcal{X}=\left\{x \mid \mathcal{L}_{c}(x) \leq \mathcal{L}_{c}\left(x_{0}\right)\right\}$. Since $\mathcal{L}_{c}$ is twice continuously differentiable on $\mathcal{X}$, condition (21) holds for some $L>0$. From (20) we define a direction $d^{k}=-\left(\bar{H}^{k}\right)^{-1} \nabla \mathcal{L}_{c}$ which is a descent direction of $\mathcal{L}_{c}$ when $\bar{H}^{k}$ is positive definite. Thus, for a sufficiently small step size $\alpha, x^{k}$ remains in the set $\mathcal{X}$ for every $k$.

As noted in [17], if the Lipschitz condition (21) holds, and if $\alpha$ is chosen such that

$$
0<\alpha<\frac{2\left|\nabla \mathcal{L}_{c}\left(x^{k}, \lambda^{k+1}\right) d^{k}\right|}{L\left\|d^{k}\right\|^{2}}
$$

then every limit point of $x_{k}$ is a stationary point of $\mathcal{L}_{c}$. Since a stationary point of $\mathcal{L}_{c}$ corresponds with a stationary point of $\mathcal{L}$, Step 2 holds.

From Step 2, the DMM converges to a stationary point of $\mathcal{L}$, and so the KKT conditions are met at this point. As argued in Step 1, KKT conditions are sufficient for global optimality, thus proving Theorem 1. Furthermore, since $N$ is full rank, the gradients of the equality constraints are linearly independent, and so all feasible points are regular. This implies that the KKT conditions are also necessary and that the optimal Lagrange multipliers (LMPs) are unique.

\section{E. Wind Model and Prediction}

To simulate wind generation, we utilize the model from [19] and generate a non-dimensional random process $w^{K}$ as $w^{K}=$ $\mathcal{H}(s, \theta) r^{K}$ where $\mathcal{H}(s, \theta)$ is a rational transfer function of order $n$ and unity DC gain, $\theta$ is a vector of parameters, and each element of $r^{K}$ is a Gaussian random variable $\mathcal{N}\left(\mu, \sigma^{2}\right)$. To normalize $w^{K}$, we use $\mu=1$ and $\sigma=1 / 3$ (see Fig. 2). The actual power produced by a wind generator is then calculated using $w^{K}$ as $P_{G r_{i}, \text { true }}^{K}=w^{K} P^{\prime}{ }_{G r_{i}}$, where $P_{G r}$ is a nominal value of the wind power. For simplicity, we assume that the same wind profile is brought to all wind generators in the system, which can occur in concentrated wind farms [20].

Since the true wind generation is not known during market clearing, the upper bound $\hat{\bar{P}}_{G r_{i}}$ from (8) must be estimated using wind prediction. Increasing penetration of wind generation has made accurate wind predictions essential to power systems operation (see [21] for a detailed overview). For short term operations, a range of statistical models have been applied to wind forecasting, including auto-regressive moving average (ARMA) models, among others [22].

We assume in this paper that $\hat{P}_{G r_{i}, \text { true }}$ can be estimated as $\hat{w}^{K} P^{\prime}{ }_{G r_{i}}$, where $\hat{w}^{K}=\mathcal{H}(s, \hat{\theta}) \hat{r}^{K}$ and $\hat{\theta}$ is an estimate of $\theta$ with a parameter error $\widetilde{\theta}$. To compute $\hat{r}^{K}$, we assume that wind conditions in the past are perfectly known, but that there is a stochastic error introduced when trying to predict future wind conditions. That is, we set

$$
\hat{r}_{D M M}^{K}=\left\{\begin{array}{cc}
r^{K} & K \in \Phi_{m-1} \\
\mathcal{N}\left(\mu, \sigma^{2}\right) & K \in \Psi_{m-1} \cup \Psi_{m}
\end{array}\right.
$$

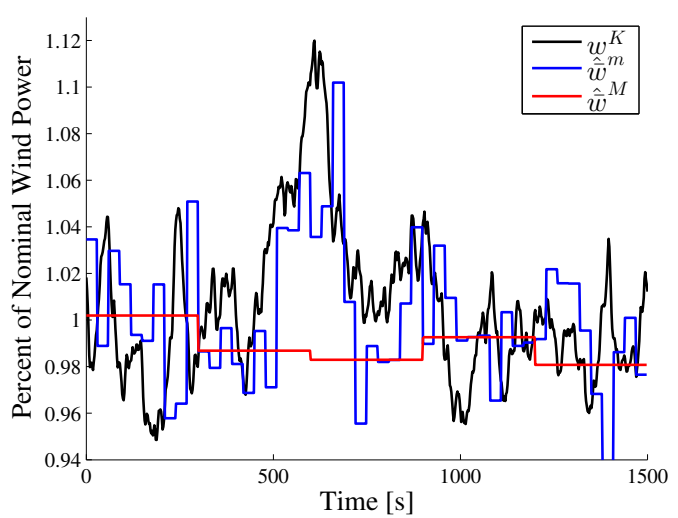

Fig. 2. True wind generation, along with sample DMM and OPF predictions.

We then determine $\hat{\bar{P}}_{G r_{i}}$ in (8) as $\hat{\bar{w}}^{m} P^{\prime}{ }_{G r_{i}}$ where $\hat{\bar{w}}^{m}$ is the average of $\hat{w}^{K}$ over $K \in \Psi_{m}$.

A similar wind prediction model can also be utilized in the OPF-method, where the estimate of $\hat{r}^{K}$ is updated at the corresponding time-scale $T_{M}$. That is, one can choose

$$
\hat{r}_{O P F}^{K}=\left\{\begin{array}{cc}
r^{K} & K \in \Phi_{M} \\
\mathcal{N}\left(\mu, \sigma^{2}\right) & K \in \Psi_{M}
\end{array}\right.
$$

and choose $\hat{\bar{P}}_{G r_{i}}$ as $\hat{\bar{w}}^{M} P^{\prime}{ }_{G r_{i}}$, where $\hat{\bar{w}}^{M}$ is the average of $\hat{w}^{K}$ over $K \in \Psi_{M}$. Fig. 2 shows an example of these prediction techniques for 25 minutes of wind power.

\section{F. Distributed Implementation of DMM}

We now discuss how the DMM in (17) and (18) can be implemented by the market players which includes the ISO, GenCos, and ConCos in a distributed manner. Each iteration of DMM requires the full update of the gradient of Lagrangian from (16) with respect to $x$ which consists of following terms

$$
\begin{aligned}
\nabla_{x} \mathcal{L}\left(x^{k}, \hat{\lambda}^{k+1}\right)= & \nabla_{x}\left(-S_{W}\left(x^{k}\right)\right)+\nabla_{x}\left(\beta_{1}\left(x^{k}\right)\right) \\
& +\nabla_{x}\left(\beta_{2}\left(x^{k}\right)\right)+N \hat{\lambda}^{k+1} .
\end{aligned}
$$

The key feature of (23) which allows distributed implementation is that each market player can evaluate corresponding terms of this equation using only its own state and its negotiated price at every iteration $k$. To show this, we analyze each of the terms in (23) individually.

First, $\nabla_{x}\left(-S_{W}\left(x^{k}\right)\right)$ is composed of cost-utility curves which belong to generators and consumers as

$$
\nabla_{x}\left(-S_{W}\left(x^{k}\right)\right)=\left[\begin{array}{c}
0 \\
-b_{D r}-c_{D r} P_{D r}^{k} \\
b_{G c}+c_{G c} P_{G c}^{k} \\
b_{G r}+c_{G r} P_{G r}^{k}
\end{array}\right] .
$$

Second, $\nabla_{x}\left(\beta_{1}\left(x^{k}\right)\right)$ is composed of barrier functions used to incorporate limits of generation and demand as

$$
\nabla_{x}\left(\beta_{1}\left(x^{k}\right)\right)=\left[\begin{array}{c}
0 \\
\nabla_{P_{D r}}\left(\beta_{1}\left(x^{k}\right)\right) \\
\nabla_{P_{G c}}\left(\beta_{1}\left(x^{k}\right)\right) \\
\nabla_{P_{G r}}\left(\beta_{1}\left(x^{k}\right)\right)
\end{array}\right] .
$$


where

$$
\nabla_{P_{\ell}}\left(\beta_{1}\right)=\frac{\nu}{\left(P_{\ell}^{k}-\bar{P}_{\ell}\right)^{2}}-\frac{\nu}{\left(P_{\ell}^{k}-\underline{P}_{\ell}\right)^{2}}
$$

for $\ell \in D_{r}, G_{c}, G_{r}$. Each row in (24) and (25) is evaluated by a corresponding generator or consumer.

Third, $\nabla_{x}\left(\beta_{2}\left(x^{k}\right)\right)$ is composed of barrier functions used to incorporate transmission system constraints as

$$
\nabla_{x}\left(\beta_{2}\left(x^{k}\right)\right)=\left[\nabla_{\delta}\left(\beta_{2}\left(x^{k}\right)\right) \quad 0 \quad 000\right]^{T}
$$

where

$$
\begin{aligned}
\nabla_{\delta_{n}}\left(\beta_{2}\left(x^{k}\right)\right)= & \sum_{m \in \Omega_{n}} \frac{\nu B_{n m}}{\left(B_{n m}\left(\delta_{n}(k)-\delta_{m}(k)\right)-\bar{P}_{n m}\right)^{2}} \\
& -\frac{\nu B_{n m}}{\left(B_{n m}\left(\delta_{n}(k)-\delta_{m}(k)\right)+\bar{P}_{n m}\right)^{2}} .
\end{aligned}
$$

The gradient in (27) is entirely evaluated by the ISO.

In order to move negotiation forward, generators and consumers must provide the gradient of their cost-utility curve including the barrier functions $\nabla_{x}\left(-S_{W}\left(x^{k}\right)+\beta_{1}\left(x^{k}\right)\right)$ for the current negotiation $x^{k}$. This represents the marginal cost of production (or marginal value of utility) for each entity at the current negotiation. The ISO evaluates $\nabla_{x}\left(\beta_{2}\left(x^{k}\right)\right)$ for the current negotiation $x^{k}$, based on the grid topology (i.e. the layout of transmission lines and the susceptances and power limits of these lines).

Additionally, each iteration of DMM requires the updated Hessian

$$
\begin{aligned}
H^{k} & =\nabla_{x x}^{2} \mathcal{L}\left(x^{k}, \lambda^{k}\right) \\
& =-\nabla_{x x}^{2} S_{W}\left(x^{k}\right)+\nabla_{x x}^{2} \beta_{1}\left(x^{k}, T\right)+\nabla_{x x}^{2} \beta_{2}\left(x^{k}\right) .
\end{aligned}
$$

Values of $\nabla_{x x}^{2}\left(\beta_{1}\right)$ and $\nabla_{x x}^{2}\left(\beta_{2}\right)$, which are needed to evaluate the Hessian, contain information about the barriers on generation and consumption which might be considered private. To compute the true Hessian $H^{k}, \nabla_{x x}^{2}\left(\beta_{1}\right)$ and $\nabla_{x x}^{2}\left(\beta_{2}\right)$ needs to be available to the ISO at every iteration. To avoid this, the Hessian is approximated as $\hat{H}$ in the following way

$$
\hat{H} \approx-\nabla_{x x}^{2} S_{W}\left(x^{k}\right)=\left[\begin{array}{cccc}
0 & 0 & 0 & 0 \\
0 & -c_{D r} & 0 & 0 \\
0 & 0 & c_{G c} & 0 \\
0 & 0 & 0 & c_{G r}
\end{array}\right]
$$

This approximation is of high accuracy when DMM states are away from the barriers, since $\nabla_{x x}^{2}\left(\beta_{1}\right) \approx 0$ and $\nabla_{x x}^{2}\left(\beta_{2}\right) \approx 0$. To avoid sharing incremental cost-utility coefficients $c_{\ell}$, we propose that the ISO use estimates for the values in $\hat{H}$, which could be a modification of the ones from the day ahead market clearing, or obtained by inference based on the type of generator or consumer. Regardless of the approach taken to obtain these coefficients, it is important to note that the equilibrium of the DMM will not be affected, but the paths that the state variables take (and hence the convergence time) will be altered.

Finally, it can be proven that the DMM converges to the global optimum of (13) for a sufficiently small step size $\alpha$ by replacing $H^{k}$ by $\hat{H}$ and following an identical series of arguments as the proof of Theorem 1 . We now require that $c$ is chosen sufficiently large to make $\hat{\bar{H}}$ positive definite.
In addition to the privacy benefit, we note that unlike $H^{k}$, the approximation $\hat{H}$ remains constant for all iterations $k$. Therefore, a single offline inversion of $\hat{H}$ is sufficient to implement the proposed market clearing mechanism, greatly reducing computational requirements.

\section{G. Accommodating Wind Intermittency}

Constraints enforced by barrier functions (6)-(8) tend to change from one market period to the next, due to changes in wind forecast, operating conditions and energy consumption throughout the day. To smoothly accommodate this change, we add another feature to the DMM to dynamically shift barrier functions in response to a change in inequality constraints.

Moving the barrier functions in a single iteration may cause $g_{1}\left(x^{k}\right)$ to become negative, but the barrier functions are only properly defined for $g_{1}\left(x^{k}\right)>0$. To avoid this scenario, the barrier functions are moved incrementally by an amount no larger than a fraction $\gamma \in(0,1)$ of the distance between current and desired position of the barrier. The algorithm for shifting an upper barrier $\bar{P}_{\ell}$ and a lower barrier $\underline{P}_{\ell}$ for a power variable $P_{\ell} \in P_{D r}, P_{G c}, P_{G r}$ can be written as

$$
\begin{aligned}
& \bar{P}_{\ell}^{k+1}=\bar{P}_{\ell}^{k}-\min \left(\gamma\left(\bar{P}_{\ell}^{k}-P_{\ell}^{k}\right), \bar{P}_{\ell}^{k}-\bar{P}_{\ell}^{m}\right) \\
& \underline{P}_{\ell}^{k+1}=\underline{P}_{\ell}^{k}+\min \left(\gamma\left(P_{\ell}^{k}-\underline{P}_{\ell}^{k}\right), \underline{P}_{\ell}^{m}-\underline{P}_{\ell}^{k}\right)
\end{aligned}
$$

where $\bar{P}_{\ell}^{k}$ and $\underline{P}_{\ell}^{k}$ are the barrier locations at iteration $k$, and $\bar{P}_{\ell}^{m}$ and $\underline{P}_{\ell}^{m}$ are the desired barrier locations of the corresponding market player for period $T_{m}$. In the case of wind generators, we set $\bar{P}_{\ell}^{m}=\hat{\bar{P}}_{G r}$.

\section{H. Clearing Intervals}

All negotiations occur at time instants $t_{k}$, whose period $T_{k}$ is assumed to be such that enough iterations can occur in a market period $T_{m}$. The assumption is that negotiations that occur between $t_{m-1}$ and $t_{m}$ converge well before $t_{m}$ and the resulting dispatch is used for operations during the period $\left[t_{m}, t_{m+1}\right]$. In other words, during the negotiation phase, each negotiation $x^{k}$ is only a potential dispatch, and is not financially binding until $t=t_{m}$. The true demand and generation are assumed to be $P_{D c_{n}, \text { true }}^{K}=P_{D c_{n}}, \forall K, P_{D r_{i}, \text { true }}^{K}=$ $\left(P_{D r_{i}}^{*}\right)^{m}, K \in \Psi_{m}$, and $P_{G c_{i}, \text { true }}^{K}=\left(P_{G c_{i}}^{*}\right)^{m}, K \in \Psi_{m}$, where $\left(P_{D r_{i}}^{*}\right)^{m}$ and $\left(P_{G c_{i}}^{*}\right)^{m}$ are the optimal values to which the DMM converges for market period $T_{m}$.

\section{INTEGRATION WITH AGC}

In this section, we address the second step of the proposed integrated DMM, which pertains to AGC. The purpose of AGC, as mentioned in the introduction, is to deal with fluctuations in load and generation that occur at a faster timescale and cannot be anticipated at the market time-scales. The typical procedure in AGC is to use grid frequency deviations from the nominal (60 Hz in the US) as an indicator of error in generation. Generator set points are then adjusted with a period $T_{K}$ (typically $2 \mathrm{sec}$ ) based on the error in frequency, which are at a much faster time-scale than those of market clearing in a real-time market, which occurs with a period $T_{M}$. In addition to the existing implementation of AGC, we 
propose a feedback algorithm for using a suitable aggregation of the error in system frequency over a period $T_{m}$ of DMM as a correction to the power balance constraint. This is discussed in more detail below.

\section{A. AGC Modeling}

For simplicity, we consider a single frequency balancing area and assume no tie lines. A simple area-level discrete time model for AGC can be written as

$$
\omega^{K+1}=\omega^{K}+\frac{T_{K}}{J_{e q}}\left(P_{G_{t o t}}^{K}-P_{D_{t o t}}^{K}-K_{R}\left(\omega^{K}-\omega_{r e f}\right)\right)
$$

where

$$
\begin{aligned}
& P_{G_{\text {tot }}}^{K}=\sum_{i \in G c} P_{G c_{i}, \text { true }}^{K}+\sum_{i \in G r} P_{G r_{i}, \text { true }}^{K} \\
& P_{D_{\text {tot }}}^{K}=\sum_{n \in N} P_{D c_{n}, \text { true }}^{K}+\sum_{i \in D r} P_{D r_{i}, \text { true }}^{K}
\end{aligned}
$$

and $K_{R}$ is the control gain for the AGC system, chosen such that the resulting closed loop, discrete time system is stable, and it is assumed that $\omega^{0}=\omega_{r e f}$. The true wind generation, $P_{G r_{i}, \text { true }}^{K}$ is due to the wind conditions at time $t_{K}$. In this paper, we assume that all conventional generators that are dispatched in the real-time market participate in providing regulation services by supplying an incremental quantity of power $P_{R_{i}}^{K}$ in response to the frequency error at each market clearing instance $t_{K}$. The amount of incremental power supplied by each regulation unit is proportional to its frequency bias factor (denoted by $B_{i}, i \in G_{c}$ ) and is equal to

$$
P_{R_{i}}^{K}=-K_{R} \frac{B_{i}}{B_{e q}}\left(\omega^{K}-\omega_{r e f}\right)
$$

where $B_{e q}=\sum_{i \in G_{c}} B_{i}$. The total power produced by all regulation units, referred to as area control error (ACE) is

$$
(A C E)^{K}=\sum_{i \in G c} P_{R_{i}}{ }^{K}=-K_{R}\left(\omega^{K}-\omega_{r e f}\right) .
$$

We introduce two performance measures to quantify the behavior of the proposed integrated DMM at the AGC timescale: regulation capacity requirement, $C_{R E G}$, and the total energy supplied by AGC, $E_{R E G}$. The regulation capacity requirement is the difference between the highest and lowest value of $A C E$ over all $K$ and is defined as

$$
C_{R E G}=\max _{K \in \tau}(A C E)^{K}-\min _{K \in \tau}(A C E)^{K}
$$

where $\tau$ is the set of all $K$ for the entire simulation. The total energy requirement (including both positive and negative components) is defined as

$$
E_{R E G}=\sum_{K \in \tau}\left|(A C E)^{K}\right| .
$$

\section{B. Feedback from $A G C$ to $D M M$}

To integrate AGC and DMM layers, any non-zero average grid frequency error that may be present is propagated from the AGC layer to the DMM layer by the means of a hierarchical feedback control loop. In this paper, we build on the feedback implementation from [14] by giving it additional and more detailed structure through the following procedure. Frequency error is aggregated over the period of a single DMM market clearing $T_{m}$ as

$$
\omega^{m}=\frac{1}{\left|\Psi_{m}\right|} \sum_{K \in \Psi_{m}} \omega^{K} .
$$

with the resulting $\omega^{m}$ is fed back into the DMM in the form of a modified power balance $h^{\prime}(x)=0$ that replaces $h(x)=0$, defined below. We note that all DMM negotiations at $t_{k} \in\left[t_{m-1}, t_{m}\right]$ use information obtained over the previous period $T_{m-1}$, and hence the aggregated frequency information available at $t_{m-1}$ corresponds to $\omega^{m-2}$. We define the modified power balance constraint as

$$
h^{\prime}\left(x^{k}\right)=h\left(x^{k}\right)-\frac{K_{L} K_{R}}{B_{e q}} A_{G c} \bar{B}\left(\omega^{m-2}-\omega_{r e f}\right)
$$

where $\bar{B}$ is a vector of elements $\bar{B}_{n}=\sum_{i \in \theta_{n}} B_{i}, \forall n \in V$ and $K_{L}$ is a feedback gain chosen by design. Intuitively, the role of vector $\bar{B}$ is to disaggregate frequency error and to distribute it to the nodes with generators who committed to provide regulation. Such implementation allows the aggregated frequency error to be met optimally by the market.

Finally, the integrated DMM consists of solving (13) with the equality constraint replaced by $h^{\prime}(x)=0$. The iterates of the integrated DMM at the time scale $t_{k}$ take the form

$$
\begin{aligned}
& x^{k+1}=x^{k}-\alpha \cdot \hat{\bar{H}}^{-1} \nabla_{x} \mathcal{L}\left(x^{k}, \hat{\lambda}^{k+1}\right) \\
& \lambda^{k+1}=\hat{\lambda}^{k+1}-\alpha \cdot c \cdot h^{\prime}\left(x^{k}\right) \\
& \hat{\lambda}^{k+1}=\left(N^{T} \hat{\bar{H}}^{-1} N\right)^{-1}\left(h^{\prime}\left(x^{k}\right)-N^{T} \hat{\bar{H}}^{-1} \nabla f\left(x^{k}\right)\right) .
\end{aligned}
$$

Eqs. (42)-(44) together with the AGC iterates (33) and AGC aggregation defined by (40) constitute the new integrated DMM proposed in this paper.

\section{DMM SIMULATION}

In this section we validate the integrated DMM described above using a modified 118 bus test case that includes wind generation and flexible consumption. The performance of the integrated DMM is evaluated both with and without feedback from AGC, and both performances are compared to that using an OPF. Comparison metrics include $e_{R E G}$ and $r_{R E G}$ (introduced in Section IV-B) at the AGC level, and the effect of underestimation of the wind at the market level.

\section{A. IEEE 118 Bus Grid}

All simulations were performed on a modified IEEE 118 bus network [23]. While the grid topology, base demand at each bus, and generator data were left unchanged from the original test files, flexible consumers and renewable generators were added according to Tables I and II. When flexible consumer $i$ is added at a bus, the base demand at that bus was decreased by $\bar{P}_{D r i}$, with $10 \%$ of the total demand chosen to be flexible. The base and incremental costs of flexible consumers are $b_{D r i}=40$ and $c_{D r i}=-0.3 \forall i$. For simplicity, we assume that demand adheres to its dispatch from the market clearing.

The total nominal capacity $P^{\prime}{ }_{G r}$ of all wind generators is chosen as $30 \%$ of total generation in the grid and scattered 
TABLE I

Addition OF FleXible CONSUMERS to 118 Bus Grid

\begin{tabular}{|l||c|c|c|c|c|c|c|c|c|}
\hline \hline Bus & 15 & 27 & 42 & 49 & 54 & 62 & 80 & 90 & 116 \\
\hline $\bar{P}_{D r_{i}}$ & 45 & 35.5 & 48 & 43.5 & 56.5 & 50 & 50 & 50 & 50 \\
\hline \hline
\end{tabular}

TABLE II

Addition of Renewable Generators to 118 Bus GRID

\begin{tabular}{|l||c|c|c|c|c|c|c|c|c|}
\hline \hline Bus & 10 & 12 & 25 & 26 & 31 & 46 & 49 & 54 & 59 \\
\hline$P^{\prime} G r_{i}$ & 170 & 150 & 145 & 230 & 150 & 200 & 190 & 290 & 200 \\
\hline \hline
\end{tabular}

throughout at buses that already contain conventional generation. The base and incremental costs of renewable generators are $b_{G r i}=1$ and $c_{G r i}=0.1 \forall i$. The frequency bias $B_{i}$ was set to 1 for all $i$. We also enforce power flow limits of 300 MW on all transmission lines so that some of the lines are congested.

It is assumed that the DMM negotiation time-scale is $T_{k}=$ $4 \mathrm{~ms}$. This enables 7500 iterations for each market clearing period and was observed to be sufficient for convergence in our tests on the 118 bus test network. An initial set of negotiations was assumed to occur so that we begin with an appropriate initial dispatch at $T_{0}$. We use $\alpha=0.001, \nu=1$ and $c=1$ in all simulations, and select our initial state variables halfway between their corresponding barrier functions; if a variable has no barrier functions, we assume it starts at zero.

\section{B. Test Cases}

We run three sets of test cases, based on the parameter error in wind prediction defined in Section II-E, with (1) $\widetilde{\theta}=0$, (2) $\widetilde{\theta}=+5 \%$, and (3) $\widetilde{\theta}=-5 \%$ respectively. In each case, for a given gain $K_{L}$, we run the DMM for 50 market clearings over a 25 minute window, for 20 different realizations of wind power $w$. Each of these 1000 runs is then repeated for five different gain values $K_{L} \in\{0,0.2,0.4,0.6,0.8\}$. Finally, over the same 25 minute window, the OPF market model is run for 20 realizations of wind power. To compare DMM and OPF we define relative performance measures $c_{R E G}=C_{R E G}^{D M M} / C_{R E G}^{O P F}$ and $e_{R E G}=E_{R E G}^{D M M} / E_{R E G}^{O P F}$.

\section{Simulation Results and Discussion}

We now outline the main results of the market simulations. We start by illustrating the ability of the DMM to rapidly schedule conventional generators and flexible consumers in response to wind predictions at the market level, which is shown in Fig. 3, through the total $P_{G c, t r u e}, P_{G r, t r u e}$, and $P_{D r, \text { true }}$ (denoted as $\mathcal{P}_{G c}, \mathcal{P}_{G r}$, and $\mathcal{P}_{D r}$ ) over a 25 minute period. It is seen that during periods of low wind generation, the DMM dispatches include higher conventional generation and lower flexible consumption. Also, during periods of high wind generation, conventional generation is lowered and flexible consumption is increased. It was also observed in this case that the DMM resulted in a more efficient utilization of the available wind in comparison with the OPF by virtue of a better prediction of $P_{G r, t r u e}$.

Next we illustrate the effects the DMM has on the AGC system in Fig. 4, where we plot the area control error over the

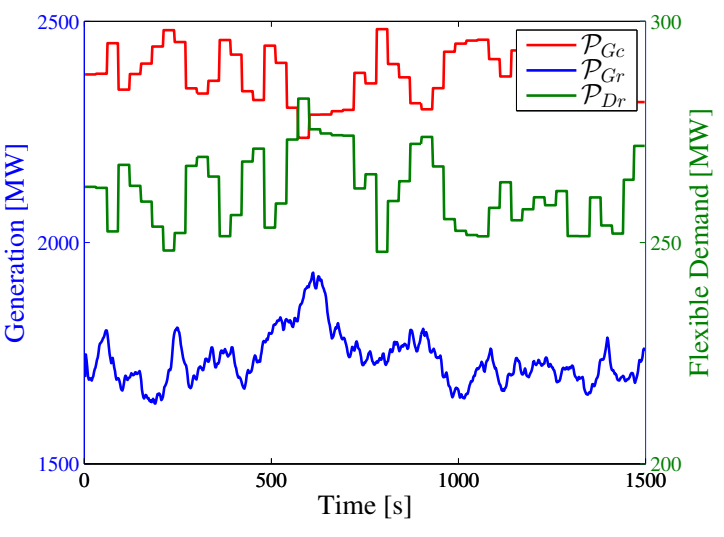

Fig. 3. Time-profile of 50 market clearings using the DMM. The iterations between any two market clearings are not shown.

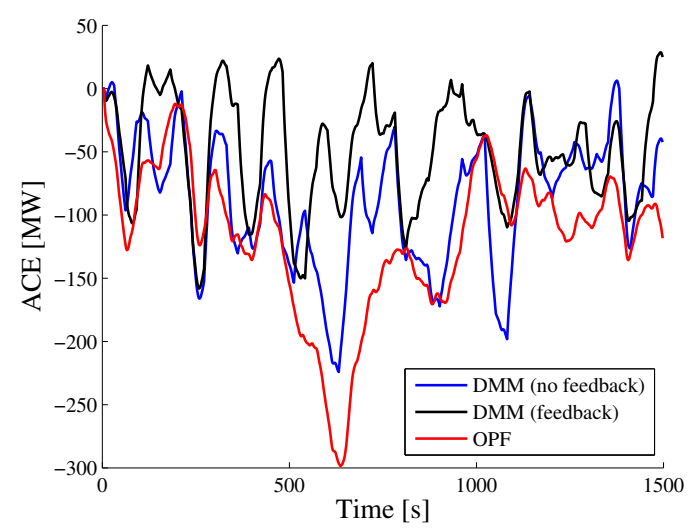

Fig. 4. ACE with the DMM and OPF for $\tilde{\theta}=-5 \%$ over a 25 minute window.

course of a 25 minute simulation with $\widetilde{\theta}=-5 \%$. Qualitatively, one can observe that the peaks in $A C E$ are less severe for the DMM simulations than the OPF. Furthermore, we get additional improvement by adding feedback from the AGC layer. This simulation included a negative parameter estimation error, which means that there was a surplus of wind energy in the grid. The feedback from AGC serves to partially correct for this surplus, shifting $A C E$ closer to zero.

Finally, we quantify these benefits and investigate the effects of the feedback gain $K_{L}$ by running the test cases. As seen in Fig. 5, for all the test cases, $e_{R E G}<1$ indicating an improvement over the OPF model. We also see that as the feedback gain $K_{L}$ is increased from 0 to $0.6, e_{R E G}$ tends to decrease even further. However, as $K_{L}$ is increased beyond $0.6, e_{R E G}$ actually starts to increase in some cases. This is because there is a delay between $t_{m-1}$, the time at which the last frequency measurement is taken, and $t_{m}$, the time at which the market dispatch is adjusted, and therefore a corresponding discrepancy in the frequency. If the feedback gain is too high, these discrepancies are amplified reducing the benefit of feedback.

From Fig. 6, it is seen that DMM also improves the regulation capacity requirements, albeit to a lesser degree. This is likely due to the stochastic nature of our prediction model. 


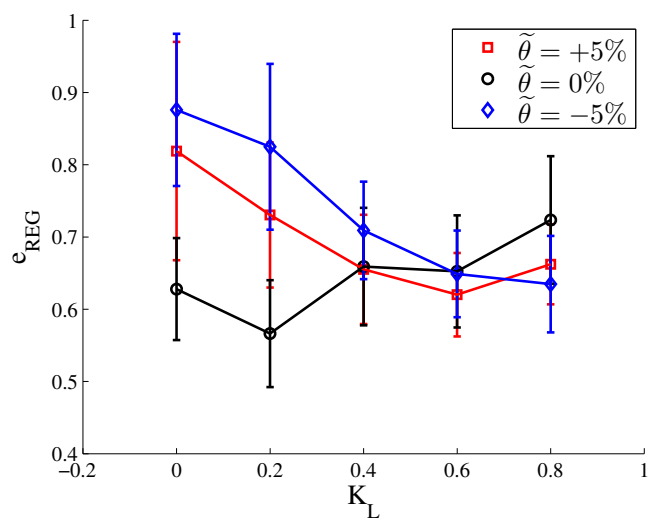

Fig. 5. Improvement in energy requirements of AGC with DMM over OPF as a function of $K_{L}$.

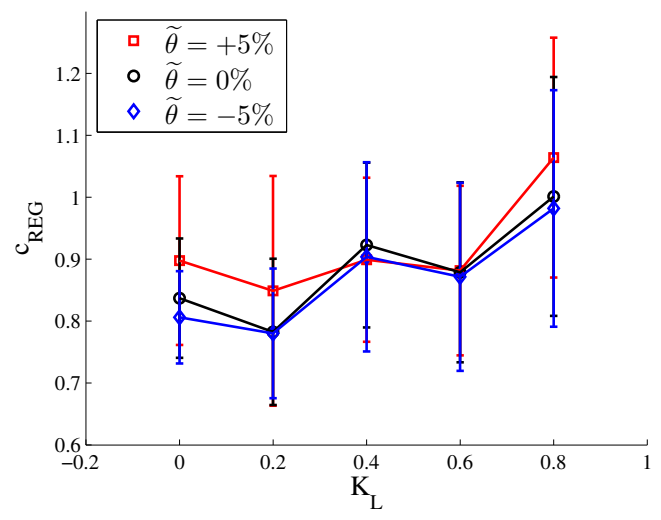

Fig. 6. Improvement in regulation requirements of AGC with DMM over $\mathrm{OPF}$ as a function of $K_{L}$.

As seen in Fig. 2, during certain market clearings there is a substantial error in the DMM prediction $\hat{\bar{w}}^{m}$. Although rare, the AGC system must be able to handle such occurrences, and thus $c_{R E G}$ is not significantly reduced. We see that $c_{R E G}$ is between 0.8 and 1.0 on average without any feedback gain. However, as the feedback gain is increased, the regulation requirement tends to increase on average.

These results suggest that perhaps there is an optimal choice for $K_{L}$, large enough to provide improvement for $e_{R E G}$, yet not so large that it results in a larger capacity requirement. In future work, we aim to model the regulation market explicitly and evaluate the reductions in energy and regulation capacity requirements in terms of a single cost saving.

\section{SUMmARY AND CONCLUDING REMARKS}

In this paper, we have described an integrated DMM which combines real-time market and frequency regulation to accommodate intermittency of RERs. We have demonstrated via simulations on the IEEE 188-bus system that (i) a NewtonRaphson-based DMM implementation enables more frequent economic dispatch than the $\mathrm{OPF}$, on the order of $30 \mathrm{sec}$ rather than $5 \mathrm{~min}$ (ii) use of an aggregate frequency error at the real-time market reduces regulation capacity requirements by about $10 \%$ and AGC energy requirements by about $25 \%$.
Additionally, we obtain these improvements while preserving the privacy of the market players' information at the real-time market. Together, these numbers demonstrate the proposed integrated DMM can serve as a viable alternative at the real-time market and AGC time-scales. How these market mechanisms can be integrated into the existing framework of planning and operations is a topic for future study.

\section{REFERENCES}

[1] B. Hodge, M. Milligan, Wind Power Forecasting Error Distributions over Multiple Timescales, IEEE PES General Meeting, 2011.

[2] H. Shun-Hsien, D. Maggio, K. McIntyre, V. Betanabhatla, J. Dumas, $\mathrm{J}$. Adams, Impact of wind generation on system operations in the deregulated environment: ERCOT experience, IEEE PES General Meeting, 2009.

[3] S. Meyn, M. Negrete-Pincetic, G. Wang, A. Kowli, E. Shafieepoorfard, The Value of Volatile Resources in Electricity Markets, IEEE Conference on Decision and Control, 49:4921-4926, 2010.

[4] H. Akhavan-Hejazi, H. Mohsenian-Rad, Optimal Operation of Independent Storage Systems in Energy and Reserve Markets with High Wind Penetration, IEEE Tran. on Smart Grid, 5(2), 2014.

[5] J. A. Taylor, D. S. Callaway, K. Poolla, Competitive Energy Storage in the Presence of Renewables, IEEE Tran. on Power Systems, 28(2), 2013.

[6] A. A. Soloman, D. M. Kammen, D. Callaway, The role of large-scale energy storage design and dispatch in the power grid: A study of very high grid penetration of variable renewable resources, Applied Energy, 134:75-89, 2014.

[7] Z. Baharlouie, M. Hashemi, H. Narimani, H. Mohsenian-Rad, Achieving Optimality and Fairness in Autonomous Demand Response: Benchmarks and Billing Mechanisms, IEEE Tran. on Smart Grid, 4(2), 2013.

[8] M. Roozbehani, M. A. Dahleh, S. K. Mitter, Volatility of Power Grids under Real-Time Pricing, IEEE Tran. on Power Systems, 27(4), 2012.

[9] P. Kundur, Power System Stability and Control, McGraw-Hill, 1997.

[10] S. Liu, M. Ilic, Hierarchical Power Systems Control, Its Value in a Changing Industry, Springer, 1996.

[11] A. Thatte, F. Zhang, L. Xie, Frequency Aware Economic Dispatch, North American Power Symposium, 2011.

[12] N. Li, L. Chen, C. Zhao, Connecting automatic generation control and economic dispatch from an optimization view, IEEE Tran. Control of Network Systems, to appear 2015.

[13] A. Kiani, A. M. Annaswamy, Equilibrium in Wholesale Energy Markets: Perturbation Analysis in the Presence of Renewables, IEEE Tran. on Smart Grid, 5(1):177-187, January 2014.

[14] A. Kiani, A. M. Annaswamy, T. Samad, A Hierarchical Transactive Control Architecture for Renewables Integration in Smart Grids: Analytical modeling and stability, IEEE Tran. on Smart Grid, Special Issue on Control Theory and Technology, 5(4):2054-2065, July 2014.

[15] A. Kiani, A. M. Annaswamy, A Dynamic Mechanism for Wholesale Energy Market: Stability and Robustness, IEEE Tran. on Smart Grid, 5(6):2877-2888, November 2014

[16] J. Hansen, J. Knudsen, A. M. Annaswamy, A Dynamic Market Mechanism for Integration of Renewables and Demand Response, submitted to IEEE Tran. on Smart Grid.

[17] D. P. Bertsekas, Nonlinear Programming, Athena Scientific, Belmont, Massachusetts, 1999.

[18] M. Ilic, J. Zaborszky, Dynamic and Control of Large Electric Power Systems, John Wiley \& Sons, 2000.

[19] A. J. Larsen, T. S. Mogensen, Individuel pitchregulering af vindmølle, M.S. Thesis, Informatics and Mathematical Modelling, Technical University of Denmark, 2006.

[20] P. E. Sørensen, N. A. Cutululis, A. Vigueras-Rodriguez, L. E. Jensen, J. Hjerrild, M. H. Donovan, H. Madsen, Power Fluctuations from Large Wind Farms, IEEE Tran. on Power Systems, 22:958-965, 2007.

[21] D. R. Chandra, M. S. Kumari, M. Sydulu, A Detailed Literature Review on Wind Forecasting, Int. Conf. on Power, Energy, and Control (ICPEC), 2013.

[22] P. Pinson, Very short-term probabilistic forecasting of wind power with generalized logit-normal distributions, Journal of the Royal Statistical Society (Applied Statistics), 61(4):555-576, 2012.

[23] Data source: https:www.ee.washington.eduresearchpstcapf118 